26 COMPARISON OF EFFECTS OF DORIS REMISSION AND LUPUS LOW DISEASE ACTIVITY STATE (LLDAS) ON DISEASE OUTCOMES IN A MULTINATIONAL PROSPECTIVE STUDY

${ }^{1}$ Eric Morand*, ${ }^{2}$ Vera Golder, ${ }^{2}$ Rangi Kandane-Rathnayake, ${ }^{3}$ Molla Huq, ${ }^{2}$ Hieu Nim, ${ }^{4}$ Worawit Louthrenoo, ${ }^{5}$ Shue Fen Luo, ${ }^{6}$ Aisha Lateef, ${ }^{7}$ Sargunan Sockalingam, ${ }^{8}$ Sandra Navarra, ${ }^{9}$ Laniyati Hamijoyo, ${ }^{10}$ Yasuhiro Katsumata, ${ }^{11}$ Madelynn Chan, ${ }^{2}$ Alberta Y Hoi, ${ }^{3}$ Mandana Nikpour. ${ }^{1}$ Monash University, Melbourne, Australia; ${ }^{2}$ Monash University; ${ }^{3}$ Melbourne University; ${ }^{4}$ Chiang Mai University Hospital; ${ }^{5}$ Chang Gung Memorial Hospital; ${ }^{6}$ National University Hospital; ${ }^{7}$ University of Malaya; ${ }^{8}$ Santo Tomas Hospital; ${ }^{9}$ Padjadjaran University; ${ }^{10}$ Tokyo Women's University; ${ }^{11}$ Tan Tock Seng Hospital

10.1136/lupus-2019-Ism.26

Background The Definitions of Remission in SLE (DORIS) group has proposed multiple definitions of remission, but these are infrequently attained and have not previously been evaluated in relation to protection from damage accrual. In contrast, the Lupus Low Disease Activity State (LLDAS) is more attainable, and has been shown to be associated with improved patient outcomes. The objective of this study was to compare the attainability and effect of LLDAS and remission on outcomes in a prospective multicenter study.

Methods A prospective multinational cohort study was undertaken in 13 centres between 2013-2017. Time dependent Cox proportional hazards models were used to compare LLDAS and DORIS definitions of remission in terms of impact on disease flares and damage accrual.

Results 1735 SLE patients were recruited, and followed for (mean \pm SD) $2.2 \pm 0.9$ years. LLDAS was achieved in 6922 visits $(54.6 \%)$. In contrast, remission was achieved in $1.1 \%-15.4 \%$ of visits. LLDAS attainment at any visit was associated with significantly reduced subsequent flare (HR 0.65, 95\% CI 0.56-0.76, p<0.001) and damage accrual (HR $0.55,95 \%$ CI $0.43-0.70, \mathrm{p}<0.001)$. In contrast, only the least stringent remission definition was associated with reduced damage accrual (HR 0.58, 95\% CI 0.39-0.88, p 0.01). Only remission definitions including serological remission were significantly associated with reduction in subsequent flares. Patients who spent $50 \%$ of their observed time in LLDAS had two-fold reduction in risk of damage accrual (HR 0.53, 95\% CI $0.41-0.68, p<0.001$ ), while only the least stringent remission definition, or the related definition excluding serology, were significantly protective against damage (HR 0.59 , 95\% CI $0.42-0.83$, p 0.003 ; HR 0.69 , 95\% CI $0.48-0.99$, p 0.05, respectively).

Conclusions LLDAS was markedly more attainable than any remission definition, whilst still conferring significant protection against flares and damage accrual. Only the least stringent remission definitions could be shown to be associated with significant reduction in damage accrual, likely reflecting a low frequency of remission attainment overall; and normal serology was required for protection from flare. LLDAS is a valid treatment target for SLE and is more achievable than remission.

Funding Source(s): The Asia Pacific Lupus Collaboration receives project support grants from UCB, GlaxoSmithKline, Janssen, Bristo-Myers Squibb, and AstraZeneca.

\section{COMBINATION TREATMENT WITH LACTOBACILLUS ACIDOPHILUS AND TACROLIMUS HAVE POTENT THERAPEUTIC EFFICACY AND IMPROVES TACROLIMUS INDUCED TH17/TREG CELL IMBALANCE IN ANIMAL MODEL OF LUPUS}

Da Som Kim*, Sung-Hwan Park, Mi-La Cho, Seung-Ki Kwok. The Catholic University

\subsection{6/lupus-2019-Ism.27}

Background Systemic lupus erythematosus (SLE) is a systemic autoimmune disease characterized by tissue-binding autoantibodies and immune complexes. Tacrolimus, also known as FK506, is an immunosuppressant that has been used for the treatment of lupus as well as for the prevention of graft rejection after organ transplantation. It achieve immunosuppressive activity by inhibiting IL-2, a molecule that promote the development and proliferation of $\mathrm{T}$ cells. However, tacrolimus induces $\mathrm{T}$ cell imbalance because IL-2 is also known to promote Treg cells and inhibit proinflammatory Th17 cells. Recently, there have been reports showing that SLE is associated with gut microbiota. Lactobacillus acidophilus, one of the typical intestinal bacteria, is reported to have therapeutic efficacy through $\mathrm{T}$ cell regulation in immune-mediated inflammatory diseases including SLE.

Methods The present study was undertaken to investigate whether combination therapy of Lactobacillus acidophilus and tacrolimus improve the therapeutic efficacy and $\mathrm{T}$ cell imbalance in animal model of SLE (MRL/lpr mice). The 8-week-old $\mathrm{MRL} / \mathrm{lpr}$ mice were orally administered with $5 \mathrm{mg} / \mathrm{kg}$ tacrolimus and/or $50 \mathrm{mg} / \mathrm{kg}$ Lactobacillus acidophilus daily.

Results The results showed that spleen size was markedly decreased in tacrolimus and Lactobacillus acidophilus combination group compared with tacrolimus alone group, and that DNT cells, which is a pathogenic immune cell subset, of MRL/ lpr mouse, were profoundly decreased in peripheral blood (PB) and spleens of mice treated with combination therapy. In addition, serum levels of ds-DNA and IgG2a were decreased, and renal pathology score was markedly alleviated by combination treatment. In vitro experiments using spleen cells from MRL/lpr mice revealed that treatment with Lactobacillus acidophilus and tacrolimus induce Treg cells and decreased Th17 cells.

Conclusions In conclusion, we demonstrated that addition of Lactobacillus acidophilus can augment the therapeutic effect of tacrolimus while improving the T cell imbalance in SLE.

Funding Source(s): None

\section{VISCERAL PSEUDO-OBSTRUCTION (VPO): A NEW TERMINOLOGY OF SYSTEMIC LUPUS ERYTHEMATOSUS GASTROINTESTINAL INVOLVEMENT AND A CT SCORING SYSTEM}

Zhiwei Chen, Jiaoyu Li, Xiaodong Wang, Ting Li, Shuang Ye*. South Campus, Ren Ji Hospital, School of Medicine, Shanghai Jiaotong University

\subsection{6/lupus-2019-Ism.28}

Background To identify the relevant parameters for SLE-VPO evaluation on computed tomography (CT), and try to develop a CT image based evaluation system for SLE-VPO. 